\title{
An Approach to Rigid Minimum Residual Power Level of Nodes in Multi-Hop Wireless Network
}

\author{
Gaurav Sharma \\ PG Scholar \\ SVITS Indore
}

\author{
Jigyasu Dubey \\ Associate Professor \\ SVITS Indore
}

\begin{abstract}
In multi hop wireless network nodes have finite battery energy to participate active role in network operations. Nodes not only dissipate amount of energy during the transmission of data even in sleep mode also. The energy dissipation of nodes arise network partition and irregular data transmission issue. To resolve that issue a lot of research has been done in which aware about battery energy of nodes during the discovery of route and data transmission. In this paper an approach is presented that specify minimum residual energy level of nodes to prevent data transmission from irregular manner. The proposed approach also offered to sending prioritized data packets and discard Route-Request packets comes from other nodes during regular transmission. The work minimized battery dissipation via discarding route discovery request packets and maximize the period of data transmissions.
\end{abstract}

\section{Keywords}

Ad-hoc Networks, Design issue, Energy efficiency, Routing protocol.

\section{INTRODUCTION}

We ask that authors follow some simple guidelines. In essence, we ask you to make your paper look exactly like this document. The easiest way to do this is simply to download the template, and replace the content with your own material.

Ad-hoc network is temporal kind of wireless networks in which nodes are operated within finite transmission range and battery power. Nodes are interact to each other when they are comes under transmission range and have sufficient battery power. Nodes not only consume battery power in active modes even they consume battery power in passive mode also. Each node in a ad-hoc networks performs the routing function for establishing communication among different nodes because it play role as a router in the networks, the "death" of even a few of the nodes due to energy exhaustion might cause disruption of service in the entire networks [1].In ad-hoc network routing procedure followed in distributed manner due to absence of centralized coordination, each node keep the routing information of its all neighbor nodes. Energy efficiency is one of the constraints to design and built ad-hoc network because every mobile node has limited energy power. The goal of energy efficiency is to improve lifetime of network and maximize network operations. The no of key features of ad-hoc network are:

\section{A. Application domain}

Ad-hoc networks design to control or handle emergency situation such as military, mobs controlling and natural disaster relief operations.
1). Infrastructure less: Each node of networks directly or indirectly (intermediate node) connected to each others. The structure of the network is not fixed means each node frequently change its geographical locations.

2). Dynamic topology: The property of nodes is to move the location impact on the topology of network. The topology is changed when nodes change respective locations.

3). Distributed Routing: Each node performs routing functions and maintain updated routing table that store route information of other nodes. Updated information of route is propagated to whole network.

\section{B. Design Issues}

During the design and build of ad-hoc network researchers face significant technical issues and challenges because of set of constraints imposed by environment.

1). MAC protocol: The nature of transmission media for adhoc network is wireless means nodes use radio signals to transmitting and receiving of data.

2). Routing protocol: The distributed nature of routing in adhoc network increases routing overheads. Absence of central coordinators every node perform route discovery procedure for communications.

3). Mobility: The mobility nature of nodes impact on the topology of network that route frequently changed and causes path breaks and network partitions problem.

4). Energy constraints: Node has limited energy to relay data packets, once battery drain out the communication is blocked and node goes into switch off mode. Nodes are in active mode and switch on if they have sufficient battery power.

5). Finite transmission range: Nodes relay packets using radio signals, the range or coverage of radio signal is limited for each node. Nodes are directly communicating to each other when they are in same range. When nodes belong different radio range then they are communicating via intermediate node those belong radio range of both node.

6). Energy Efficiency: This is particularly true if a node runs out of battery energy and unable to forward any messages, it effectively falls out of the Network [2]. In this case, the route breaks and AODV finds an alternate route via another route discovery. However, nodes dying can adversely affect the operational life time of Ad-Hoc Network. The dying nodes are not the communication end points, then Network connectivity will become sparser and Network partition becomes more likely. The goal of proposed method is routing or re-routing around nodes low on battery power as far as possible. However, this should be done in such a way that other useful performance metrics (e.g., end-to-end delay and Throughput) are not compromised in a significant way. 


\section{Energy Consumption Scenario}

Several studies have dealt with measuring energy consumption in the wireless interfaces of mobile nodes to determine the exact sources of energy consumption in the wireless interfaces [3]. These studies examined the different modes of operation of the wireless interfaces. It was found that a mobile node's wireless interface consumes energy not only while communicating with other nodes, but also while in idle mode, i.e. when the node is listening to the medium but not handling packets. Following are the types of energy consumption that have been identified.

Energy consumed while sending a packet

Energy consumed while receiving a packet

Energy consumed while in idle mode

Energy consumed while in sleep mode when the mobile node is turned off.

1). Energy Consumption Overhead: As indicated above, energy consumption through communications in MANET is caused by several sources. Some of these sources are useful while others are considered as a waste that should be eliminated or reduced in future designs for energy efficient schemes. Some of the main sources of wasted energy in wireless interfaces can be attributed to the following [4].

2). Idle Condition: With interfaces sitting idle most of the time especially in applications such as e-mail or webbrowsing.

3). Collisions: In this situations, which happen mainly at high load conditions, data involved in the collision become useless and the energy used to communicate this data is lost.

4). Protocol Overhead: This refers to protocol-specific control messages which impose additional energy requirements on top of what is necessary to transmit payload traffic. Managing this source adds a design requirement to MANET targeted protocols.

5). Energy efficiency metrics: Energy efficiency metrics are needed to evaluate energy conservation schemes. Energy consumed during node communications will be focused on some studies, such as metrics for energy efficiency in AdHoc Networks [5]. Several distinct metrics can be used to compare the energy-efficiency of a Routing Protocol. The selection of appropriate metrics depends on the overall goal of the Ad-Hoc Network deployment. Examples of the metrics that are used to manage energy consumption are:

Minimize energy consumed/packet

Maximize time to Network partition

Minimize variance in node energy levels

Minimize cost/packet

Minimize maximum node cost

\section{ROUTING PROTOCOL}

Ad-hoc networks are self-organizing and self-configuring multi-hop Wireless Networks, where the structure of the Network changes dynamically [6]. This is mainly due to the mobility of the nodes. In mobile Ad-Hoc Networks there is no infrastructure support as is the case with Wireless Networks, and since a destination node might be out of range of a source node transferring packets, so there is need of a routing procedure. Procedures always ready to find a path so as to forward the packets appropriately between the source and the destination. Routing Protocols in mobile ad-hoc network can be classified in many forms, but most of these are done depending on routing strategy and network structure [7]. The Routing Protocols can be categorized as flat routing, hierarchical routing and geographic position assisted routing while depending on the Network structure. According to the routing strategy Routing Protocols can be classified as tabledriven and source initiated.

\section{A. Pro-Active or Table Driven Routing Protocols}

Proactive MANET protocols are also called as table-driven protocols and will actively determine the layout of the Network. [7]. Proactive MANET protocols work best in Networks that have low node mobility or where the nodes transmit data frequently. Examples of Proactive MANET Protocols include:

Optimized Link State Routing (OLSR)

Destination-Sequenced Distance Vector (DSDV)

Cluster-head Gateway Switch Routing Protocol (CGSR)

\section{B. Reactive or On Demand Protocols}

This kind of protocols is usually based on flooding the Network with Route Request (RREQ) and Route reply (RERP) messages .By the help of Route request message the route is discovered from source to target node and as the target node gets a RREQ message it send RERP message for the confirmation that the route has been established. The different types of On Demand driven protocols are:

Ad-Hoc on Demand Distance Vector (AODV)

Dynamic Source Routing Protocol (DSR)

Temporally ordered routing algorithm (TORA)

Signal Stability-Based Adaptive Routing (SSA)

Location-Aided Routing Protocol (LAR)

\section{LITERATURE SURVEY}

Several approaches have been developed to address the energy efficiency issues in Ad-Hoc Networks. These techniques differ in the methodology as well as the layer of the protocol stack at which they function [8]. Algorithms belonging to the category may operate at the MAC layer level, Network layer level, or in between. The following section covers the most significant algorithms that belong to these categories.

\section{A. Power-Aware Routing}

Authors explore power-aware metrics to use with Routing Protocols on top of their MAC power savings protocol, PAMAS [9]. They indicate that the strategy followed by the different Routing Protocols that are not power conscious would lead to fast depletion of battery power and hence quick degradation of the Network operation. We have already discussed these metrics. The authors implemented the first and fourth metrics (minimize energy consumed per packet and minimize cost per packet, respectively). In their simulations, the authors used sparsely populated Networks and they did not consider mobility in their simulations. The reason behind not using mobility is that the evaluation is done for power management and not routing. In our view, mobility has a considerable effect on the performance of power efficient mechanisms. 


\section{B. Maximum Battery Life Routing}

A power-aware Routing Protocol that distributes power consumption evenly over nodes and minimizes the overall transmission power is proposed in [10]. This protocol uses the $\gamma$ conditional max-min battery capacity routing (CMMBCR) scheme. It uses battery capacity instead of a cost function as a route selection metric. When all nodes on some possible routes between a source and a destination have sufficient remaining energy above a certain value, $\gamma$, the route with the minimum total transmission power (MTRP) among these routes is chosen. If all routes have nodes with low battery capacity, routes that include nodes with the lowest battery capacity should be avoided to extend the lifetime of the nodes. If the value of $\gamma$ is zero, the CMMCBR reduces to MTRP. If the value of $\gamma$ is equal to the maximum (100), the CMMBCR scheme reduces to the Min-Max battery cost routing (MMBCR) scheme.

\section{Analysis of Energy Efficient Routing Techniques}

The main focus of research on Routing Protocols in MANETs has been Network performance [11]. There has been some study on Energy aware Routing Protocols for MANETs. Presented below is a brief review of some of them.

\section{Minimum Energy Routing}

Author proposes a routing algorithm based on minimizing the amount of energy per bit required to get a packet from source to destination [12]. This link cost can be defined for two cases. When the transmit energy is fixed. When the transmit energy is varied dynamically as a function of the distance between the transmitter and intended receiver.

\section{PROPOSED APPROACH}

The objective of proposed work is improve transmission periods of nodes via specifying minimum residual energy level of nodes to prevent data transmission from irregular manner. The proposed approach also offered to sending prioritized data packets and discard Route Request packets comes from other nodes during regular transmission. The work minimized battery dissipation via discarding route discovery request packets and maximize the period of data transmissions. The complete working of the approach is defined in below steps and work flow.

Step1- Initially nodes are charged with battery power $\{\mathrm{E}=100\}$

Step2-Set two threshold values for prioritize data transmission and alerting $\{\mathrm{TV} 1=25$ and $\mathrm{TV} 2=1\}$

Step3-Verify battery power of nodes and taken some actions during the routing procedures.

If battery power is much than first threshold value $\{E>T V 1\}$ then nodes send regular data packets.

Else If battery power is less than equal to first threshold value and much than second threshold value $\{E<=25$ and $E>1\}$ then nodes send prioritize data packets and discard route request packets from other nodes.

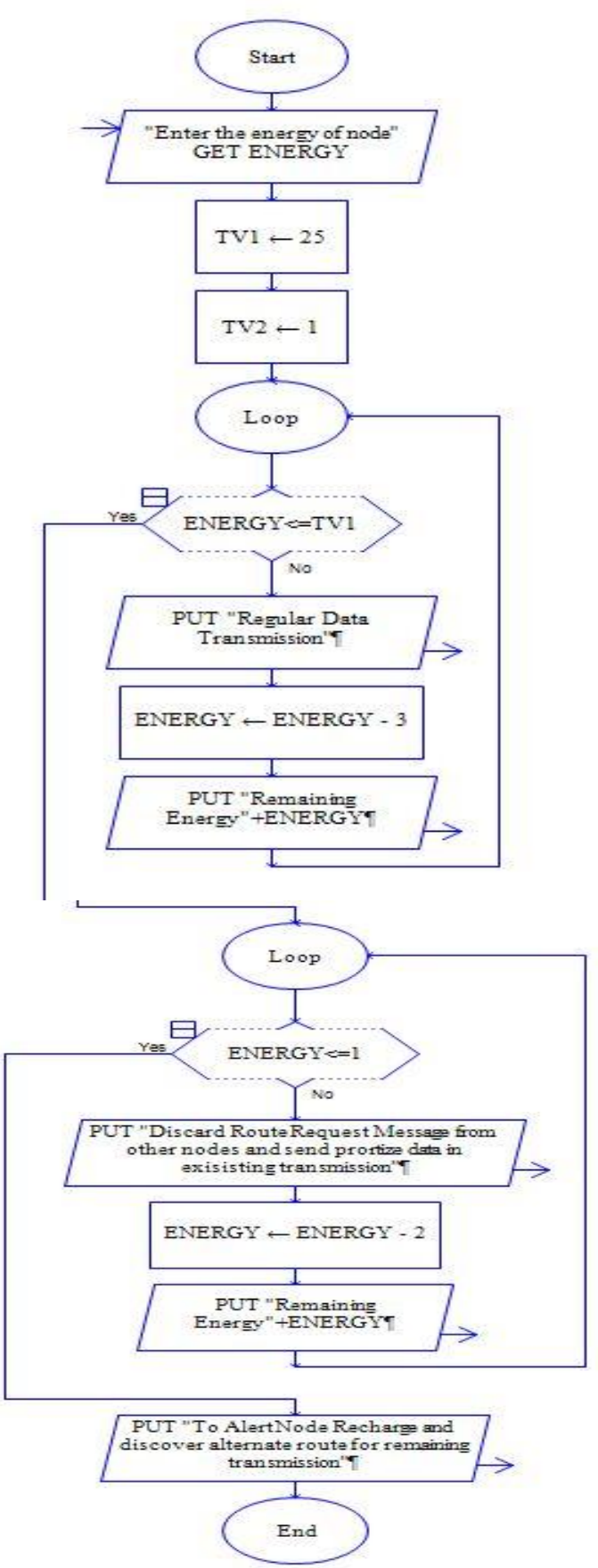

Figure.1 Work-Flow of Proposed Approach

\section{SIMULATION \& RESULTS ANALYSIS}

Proposed work simulated and obtained result analysed considering different parameters that outlined in Table-1. 
Table-1 Simulation Parameter

\begin{tabular}{|l|c|}
\hline Number of nodes & 50 \\
\hline Dimension of simulated area & $800 \times 600$ \\
\hline Initial node energy (joules) & 100 \\
\hline Threshold value(joule) & 25 \\
\hline Minimum threshold value(joule) & 1 \\
\hline Simulation time (seconds) & 150 \\
\hline Radio range & $250 \mathrm{~m}$ \\
\hline Traffic type & CBR, \\
\hline Packet size (bytes) & 512 \\
\hline Number of traffic connections & 4,30 \\
\hline Maximum Speed (m/s) & 35 \\
\hline Node movement & Random \\
\hline Transmission energy consumption & $1.0 \mathrm{~J}$ \\
\hline Receiving energy consumption & $0.75 \mathrm{~J}$ \\
\hline Idle energy consumption & $0.017 \mathrm{~J}$ \\
\hline Sleeping energy consumption & $0.001 \mathrm{~J}$ \\
\hline
\end{tabular}

\section{A. Simulation Scenario}

The overall goal of the simulation experiments is to measure energy level accuracy and increasing life time for wireless mobile ad hoc networks while continuing to successfully deliver data packets to their destinations.

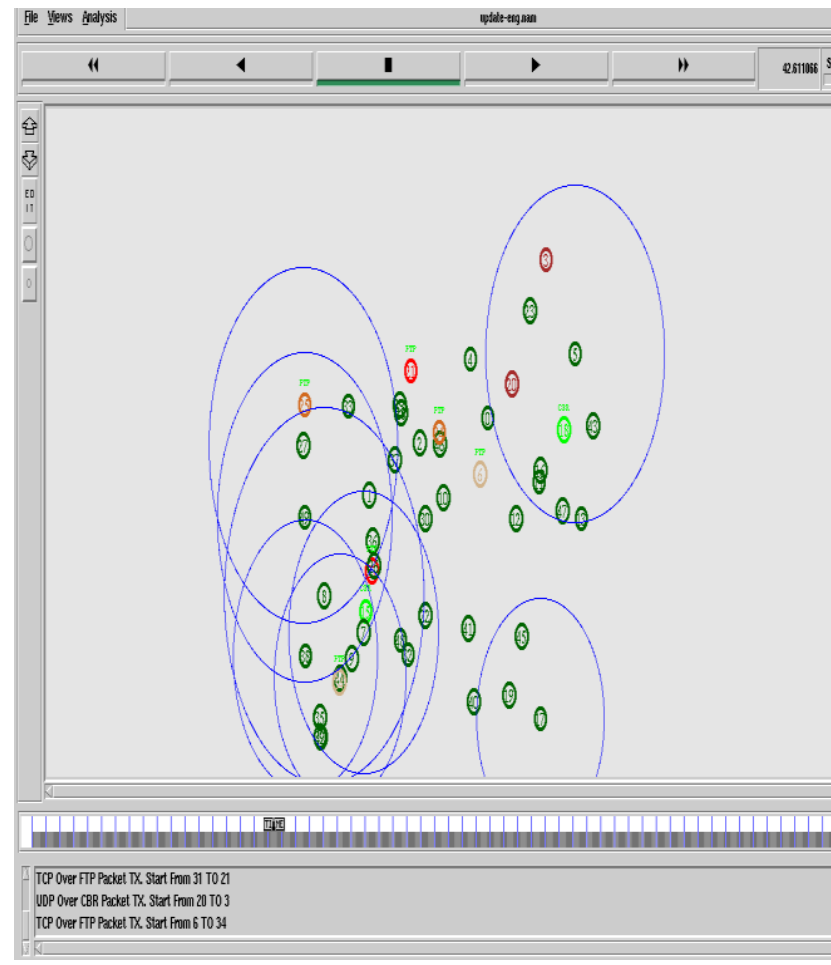

Figure.2 Simulation Scenario

\section{B. Energy Consumption Rate of Existing Approach}

The graph represents the random energy level of different nodes in simulation time $150 \mathrm{sec}$. In the graph clearly shown that about in $100 \mathrm{sec}$ time all remaining nodes are lost their energy. It means no node in the network will take part in communication after $100 \mathrm{sec}$. so according to that graph network life time is nearly 100 second only but simulation time is 150 second

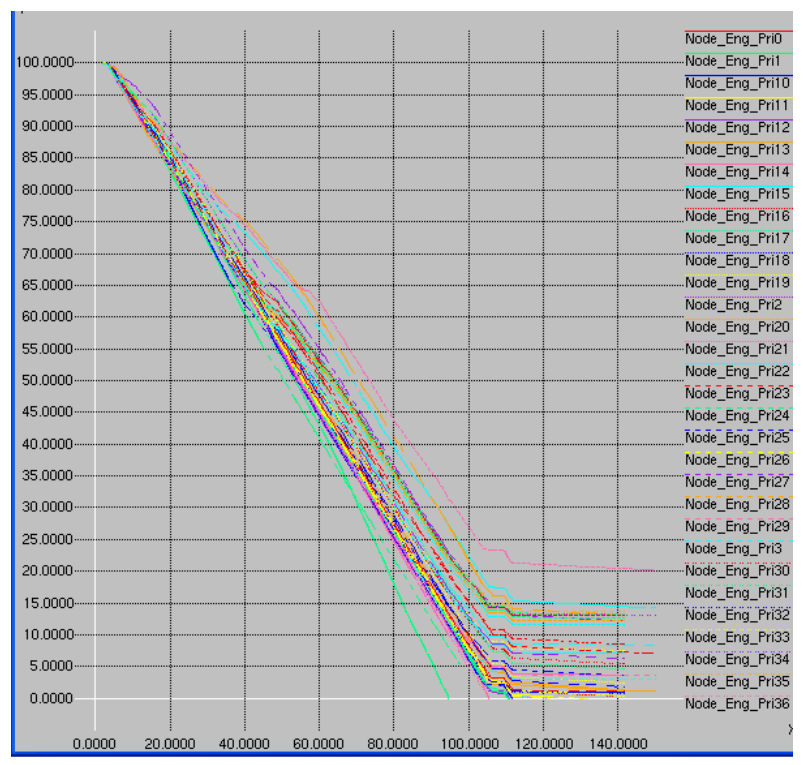

\section{Figure.3 Energy Consumption Rate of Existing Approach}

\section{Energy Consumption Rate of Proposed Approach}

In the graph clearly shown that about in $140 \mathrm{sec}$ time all remaining nodes have left their energy. It means nodes in the network will take part in communication after $100 \mathrm{sec}$. so according to that graph network life time is nearly 140 second where simulation time is 150 second. The proposed approach increases transmission periods and network lifetime as existing approach.

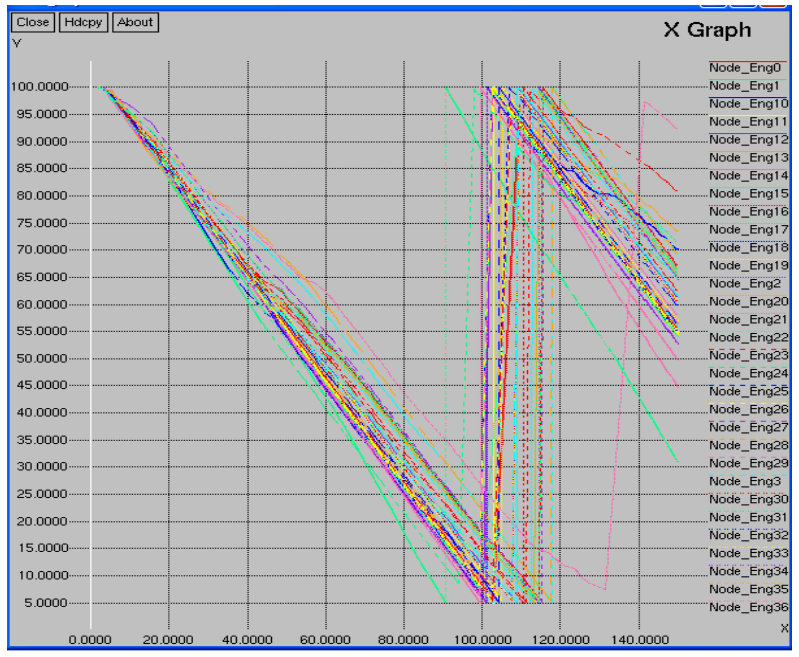

Figure.4 Energy Consumption Rate of Proposed Approach 


\section{CONCLUSION}

Node energy is precious resources in mobile ad hoc network, network operation periods affected by power dissipation ratio of nodes. Battery power consumption depends on load of nodes because intermediate nodes work as routers which are receive data from downstream and send to upstream nodes. So this reason battery power of intermediate nodes is drained out earlier as terminal nodes. The power of intermediate nodes is drained out earlier then network partitioned and short communication periods occurred. Proposed approach minimized the dissipation of battery power of nodes that enables nodes to send prior data and alert about power status of nodes to discover new alternative ways. Proposed approach improved communication periods and minimize network partitioned.

\section{ACKNOWLEDGMENTS}

I would like to thank God, my Anchor and my Reason for being, for the talent and the energy he gave me to complete this work.

I owe special thanks to my esteemed guide Mr. Jigyasu Dubey, Assistant Professor in the Department of Computer Science \& Engineering, Shri Vaishnav Institute of Technology and Science Indore, to involving right from the inception of ideas to the finalization of the work. I am deeply indebted to him. His help, suggestions and encouragement helped me in all the time of research work, and for writing of this thesis. I learned many things from him.

\section{REFERENCES}

[1] Morteza Maleki, Karthik Dantu, and Massoud Pedram, Power-aware Source Routing Protocol for Mobile Ad Hoc Networks, Dept. of EE-Systems, University of Southern California, Los Angeles, CA 90089.

[2] B. Soujanya, T. Sitamahalakshmi, C. Divakar, "Study of Routing Protocol in MANET," International Journal of Engineering Science and Technology, Vol. 3, No. 4, pp. 2622-2631, 2011.

[3] D. Nitnaware, A. Verma, "Performance Evaluation of Energy Consumption of Reactive Protocols under SelfSimilar Traffic," International Journal of Computer Science \& Communication, Vol. 1, No. 1, pp. 67-71, 2010
[4] M. Fotino, F. Rango, "Energy Issues and Energy Aware Routing in Wireless Ad-hoc Networks," INTEC Open Access publisher of scientific Books and Research, University of Calabria Italy, Vol. 1, No. 1, pp. 156-167, 2011.

[5] W. Hinzelman, A. Chandrakasan, H. Balakrishnan, "Energy-Efficient Communication Protocol for Wireless Microsensor Networks," Hawaii International Conference on System Sciences, pp. 98-106, 2000

[6] P. Misra, "Routing Protocols for Ad Hoc Mobile Wireless Networks," http://www.cis.ohiostate.edu/ jain/cis788-99/adhoc_routing/index.html, pp 234-244, 2006

[7] B. Maqbool, M. Peer, "Classification of Current Routing Protocols for Ad Hoc Networks - A Review," International Journal of Computer Applications, Vol. 7, No. 8, pp. 46-55, 2010

[8] N. Vassileva, F. Barcelo, "A Survey of Routing Protocols for Maximizing the Lifetime of Ad Hoc Wireless Networks," International Journal of Software Engineering and Its Applications, Vol. 2, No. 3, pp. 7789, 2008

[9] S. Singh, M. Woo, C. Raghavendra, "Power-aware Routing in Mobile Ad Hoc Networks," Proceedings of the ACM Mobile Computing and Networking Conference, Dallas, Texas, pp. 181-190, 1998

[10] C. Toh, "Maximum Battery Life Routing to Support Ubiquitous Mobile Computing in Wireless Ad Hoc Networks," IEEE Communications Magazine, pp. 2-11, 2001

[11] L. M. Feeney and M. Nilsson, "Investigating the Energy Consumption of a Wireless Network Interface in an $\mathrm{Ad}$ Hoc Networking Environment," Proceedings of IEEE INFOCOM 2001, Vol. 3, Anchorage AK, pp. 1548-1557, 2001

[12] V. Rishiwal, M. Yadav, S. Verma, S. K. Bajapai, "Power Aware Routing in Ad Hoc Wireless Networks, Journal of Computer Science and Technology," Vol. 9, No. 2, pp. 101-109, 2009 